\title{
Relationship between Heterogeneity of Insulin Responses and Insulin Resistance in Normal Subjects and Patients with Chemical Diabetes*
}

\author{
G. M. Reaven ${ }^{1}$ and J. M. Olefsky ${ }^{2}$ \\ Department of Medicine, Stanford University School of Medicine and Veterans Administration Hospital, Palo Alto, California, USA
}

Summary. Plasma insulin responses and insulin resistance were determined in 75 subjects, defined as having a normal, borderline abnormal, or abnormal oral glucose tolerance test (OGTT). Although considerable heterogeneity of insulin response existed, most patients with abnormal OGTT's had insulin responses greater than normal; none had insulin responses less than normal. The degree of insulin resistance also varied, but most patients with abnormal OGTT's were also abnormally insulin resistant. A significant correlation $(\mathrm{r}=0.64, \mathrm{p} \pm 0.001)$ existed between insulin response and the degree of insulin resistance. However, when both variables were taken into consideration, the entire population could be divided into two groups. One group was characterized by both normal insulin responsiveness and sensitivity, the other by increased insulin response, associated with greater insulin resistance. Most patients with abnormal OGTT's fell into the latter group, but some had glucose intolerance without either an exaggerated insulin response or insulin resistance. These results suggest that true heterogeneity exists in patients with abnormal OGTT's.

Key words: Insulin resistance, insulin response, maturity onset diabetes, Aetiology of diabetes, Heterogeneity of diabetes.

\footnotetext{
* This work was supported in part by grants from the National Institutes of Health, \#HL 08506 from the National Heart and Lung Institute and \#RR-70 from the General Clinical Research Centers Branch, and from the Research Services of the Veterans Administration

1 Dr. Reaven is a Medical Investigator, Veterans Administration

2 Dr. Olefsky is a Clinical Investigator, Veterans Administration
}

Fajans and associates have recently drawn attention to the heterogeneity of insulin responses in normal subjects and in patients with chemical diabetes [1], and have divided patients with comparable degrees of glucose intolerance into "low" and "high" insulin responders. This observation is of importance in any formulation of the pathogenesis of diabetes mellitus, and efforts should be made to define the significance of this heterogeneity of insulin response in patients with mild diabetes. Many patients with mild diabetes mellitus are more resistant to the action of insulin than normoglycaemic subjects [2], and it is possible that heterogeneity of insulin response and insulin resistance are related. To explore this possibility we have separately measured insulin response and insulin resistance in 75 non-obese subjects, classified as normal, borderline glucose intolerant, or chemically diabetic on the basis of an oral glucose tolerance test (OGTT).

\section{Methods}

The data to be presented have been derived from investigation of 75 individuals who volunteered to take part in our advertised studies of the aetiology of diabetes. Their stated reasons for participation included a family history of diabetes, a desire to have a thorough physical examination, a wish to be part of a research programme, etc. Following discussion of the nature of the protocol and after consenting to be part of the study, all subjects were admitted to the Stanford Clinical Research Centre. None of the subjects had ever received insulin, not were they taking medications known to modify carbohydrate metabolism. There was no clinical or laboratory evidence of hepatic or cardiovascular disease. The subjects were fed a liquid, weight-maintenance, formula 
Table 1. Diagnostic criteria

\begin{tabular}{llll}
\hline Group & \multicolumn{3}{l}{ Plasma glucose concentration $(\mathrm{mg} / 100 \mathrm{ml})$} \\
\cline { 2 - 4 } & Fasting & One hour & Two hour \\
\hline Normal & $<110$ & $<165$ & $<135$ \\
Borderline tolerance & $<110$ & $165-185^{\mathrm{a}}$ & $135-140^{\mathrm{a}}$ \\
Chemical diabetes & $<110$ & $>185$ & $>140$ \\
\hline
\end{tabular}

a Either one or both of the one and two hour plasma glucose values are within these ranges

Table 2. Clinical characteristics

\begin{tabular}{|c|c|c|c|c|}
\hline Group & $\begin{array}{l}\text { Num- } \\
\text { ber }\end{array}$ & $\begin{array}{l}\text { Mean }( \pm S D) \\
\text { Age y }\end{array}$ & Sex & $\begin{array}{l}\text { Mean }( \pm S E) \\
\text { Relative Weight }{ }^{\mathrm{a}}\end{array}$ \\
\hline $\begin{array}{l}\text { Normal } \\
\text { Borderline }\end{array}$ & 25 & $44 \pm 11$ & $21 \mathrm{M}: 4 \mathrm{~F}$ & $1.03 \pm .02$ \\
\hline $\begin{array}{l}\text { tolerance } \\
\text { Chemical } \\
\text { diabetes }\end{array}$ & 25 & $\begin{array}{l}47 \pm 10 \\
47 \pm 11\end{array}$ & $\begin{array}{l}20 \mathrm{M}: 5 \mathrm{~F} \\
22 \mathrm{M}: 3 \mathrm{~F}\end{array}$ & $\begin{array}{l}1.04 \pm .02 \\
1.05 \pm .02\end{array}$ \\
\hline
\end{tabular}

a Actual weight divided by ideal weight (as determined by Metropolitan Life Insurance Tables)

diet, consisting of $43 \%$ carbohydrate, $42 \%$ fat, and $15 \%$ protein, three times daily in portions of one-fifth, two-fifths and two-fifths of total calories, respectively. Daily weights were obtained; significant weight changes did not occur during the study period.

After at least three days on the diet, subjects were given an oral glucose load of 40 gm per sq. metre body surface area, and blood samples were drawn during the following three hours for measurement of plasma glucose and immunoreactive insulin levels. Subjects were divided into three groups on the basis of their plasma glucose responses during the OGTT, using the criteria given in Table 1 . The results to be reported are based upon study of 25 consecutive patients in each of the three diagnostic categories. The clinical characteristics of the three groups are presented in Table 2.

Two days later, after a $12 \mathrm{~h}$ overnight fast, all subjects received an intravenous injection of $5 \mathrm{mg}$ of propranolol followed, in five minutes, by a constant intravenous infusion of glucose $(6 \mathrm{mg} / \mathrm{kg} / \mathrm{m})$, insulin (80 $\mathrm{mU} / \mathrm{min})$, epinephrine $(6 \mu \mathrm{g} / \mathrm{min})$ and propanolol $(0.08 \mathrm{mg} / \mathrm{min})$. The solution was infused continuously for 150 minutes via a Harvard pump (Harvard Apparatus Co., Millis, Mass.) into an antecubital vein. Blood samples were drawn from the opposite arm through a needle kept patent by a slow saline infusion. Under these experimental conditions, endogenous insulin secretion is suppressed and steady state plasma glucose (SSPG) and insulin
(SSPI) levels are reached by 90 minutes [2, 3]. Studies in which the coefficients of variation of the individual SSPG and SSPI values are greater than $10 \%$ are excluded from analysis. Since similar SSPI values were achieved in all subjects [2, 3], this technique allows us to compare the ability of different subjects to dispose of identical glucose loads under the same insulin stimulus. Therefore, the mean of several plasma glucose concentrations measured during the steady state period from 90 to $150 \mathrm{~m}$ is a measure of efficiency of insulin-mediated glucose utilization, i. e. insulin resistance.

Blood for determination of plasma glucose and insulin was drawn into test tubes containing EDTA, the plasma quickly separated, and aliquots stored at $-20^{\circ} \mathrm{C}$ until determinations were done. Plasma glucose was measured by methods modified for use on a Technicon Auto Analyzer (Technicon Instruments Corp., Tarrytown, N.Y.). Plasma insulin was measured by the method of Desbuquois and Aurbach [4]. Statistical analysis was performed with Student's t-test for nonpaired data.

\section{Results}

Mean plasma glucose and insulin responses of the three groups of patients are presented in Figure 1. Despite higher mean plasma glucose levels, there was no difference in the insulin response of patients with borderline glucose tolerance, compared with normal subjects. The mean plasma insulin level of patients with chemical diabetes was never less than normal, and was significantly elevated at 1,2 and $3 \mathrm{~h}(\mathrm{p}<0.02-\mathrm{p}<0.001)$ in the face of marked hyperglycaemia.

The fact that the mean insulin response of patients with chemical diabetes was actually greater than that of normal subjects indicates that the abnormal glucose tolerance in these patients cannot be simply attributed to insulin deficiency. On the other hand, these data do not mean that all patients with an abnormal OGTT have an elevated insulin response, and inspection of the magnitude of the standard errors of the mean insulin response (Fig. 1) suggests considerable variability. In order to illustrate more clearly the degree of this variability, we have computed total insulin response (area under the insulin response curve during the OGTT) for each subject. These data are plotted in Figure 2 and indicate that the coefficient of variation of the insulin response was $23 \%, 35 \%$ and $45 \%$ for normal subjects, subjects with borderline tolerance and with chemical diabetes, respectively. However, in spite of this degree of variation, most patients with chemical 


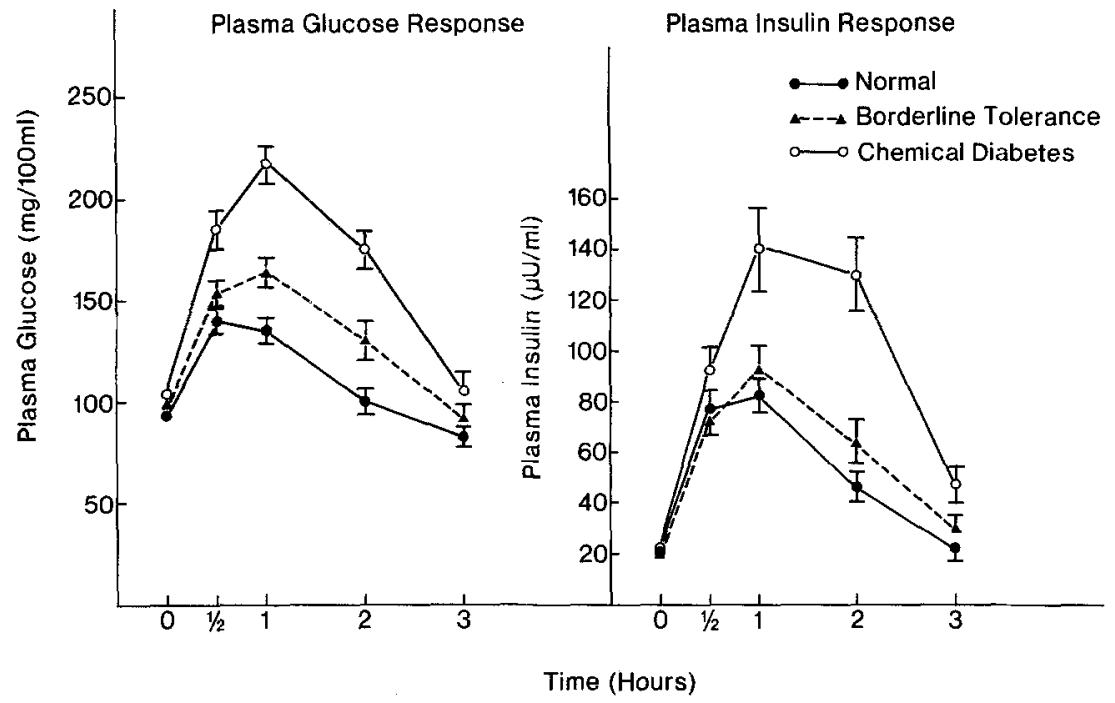

Fig. 1. Mean ( $\pm \mathrm{SE})$ plasma glucose and insulin responses during the oral glucose tolerance test (OGTT) in the three groups of subjects. There are 25 patients in each diagnostic category

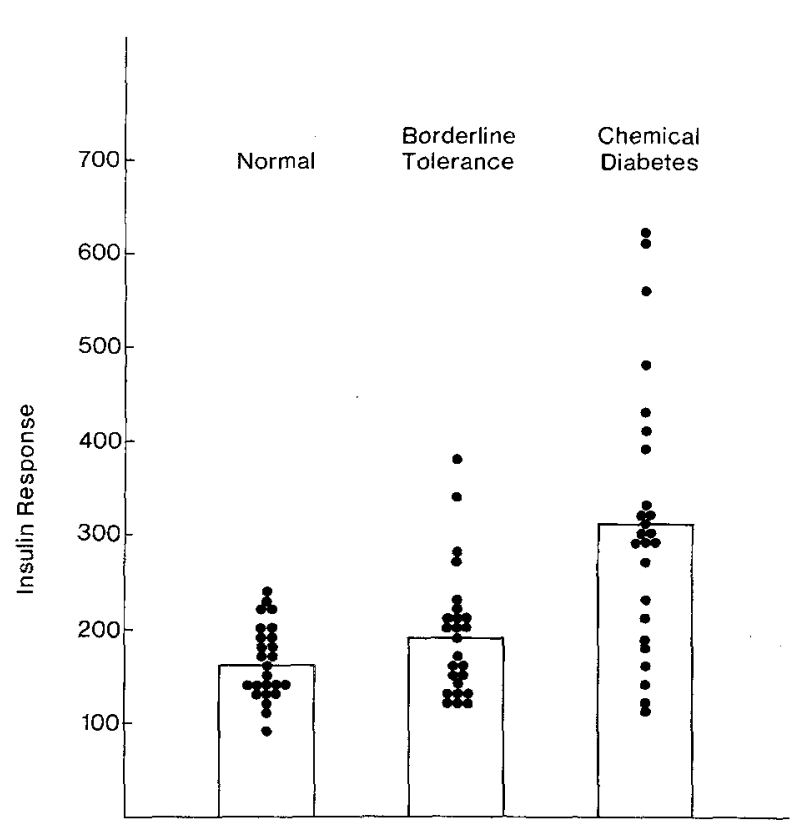

Fig. 2. Individual insulin responses (area under the plasma insulin curve) during the OGTT in each group of subjects. The mean response of each group is given by the height of the bar

diabetes had insulin responses which were greater than those of the controls. Thus, of twenty-five patients with chemical diabetes, seventeen had a total insulin response greater than that of any normal subject and eight had insulin responses within the normal range. Four patients with borderline tolerance had insulin responses greater than those of the normal subjects, while the remainder were within the normal range. In view of these results it is difficult to attribute the glucose intolerance of most patients with chemical diabetes to deficient insulin secretion.

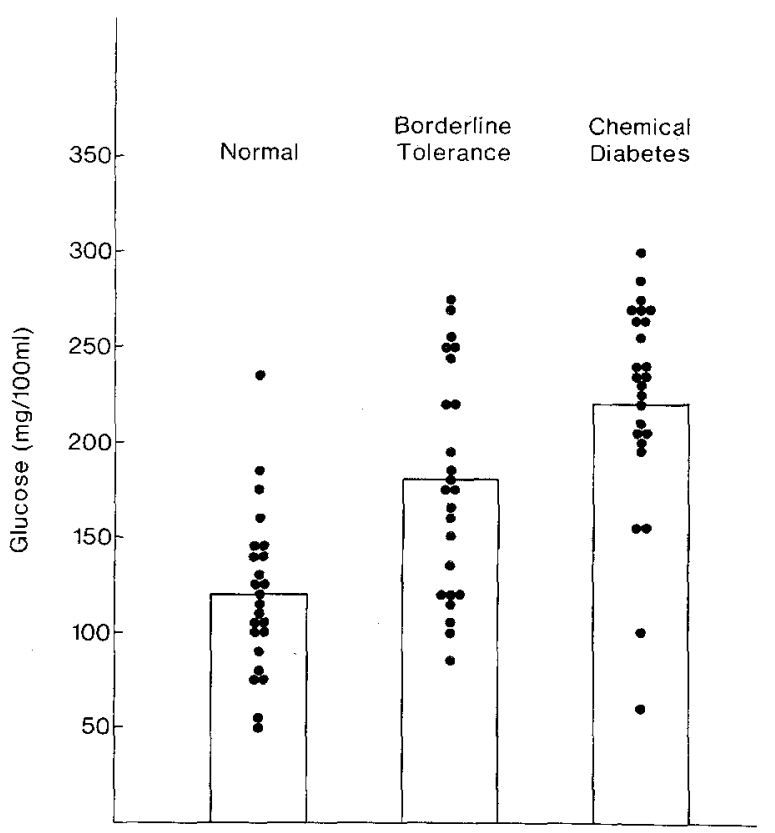

Fig. 3. Individual steady state plasma glucose responses during the infusion of epinephrine, propranolol, glucose and exogenous insulin. The mean response of each group is given by the height of the bax

An alternative explanation for the glucose intolerance of these patients is that they are resistant to the action of insulin; our attempt to quantify this resistance is seen in Figure 3, which depicts both the mean steady state plasma glucose (SSPG) responses to the infusion of glucose, insulin, epinephrine and propranolol, as well as the individual values. The mean SSPG responses are significantly $(p<0.001)$ higher than normal in patients with borderline tolerance and chemical diabetes. It should also be noted that the mean SSPG response was significantly higher in the subjects with chemical diabetes than in pa- 


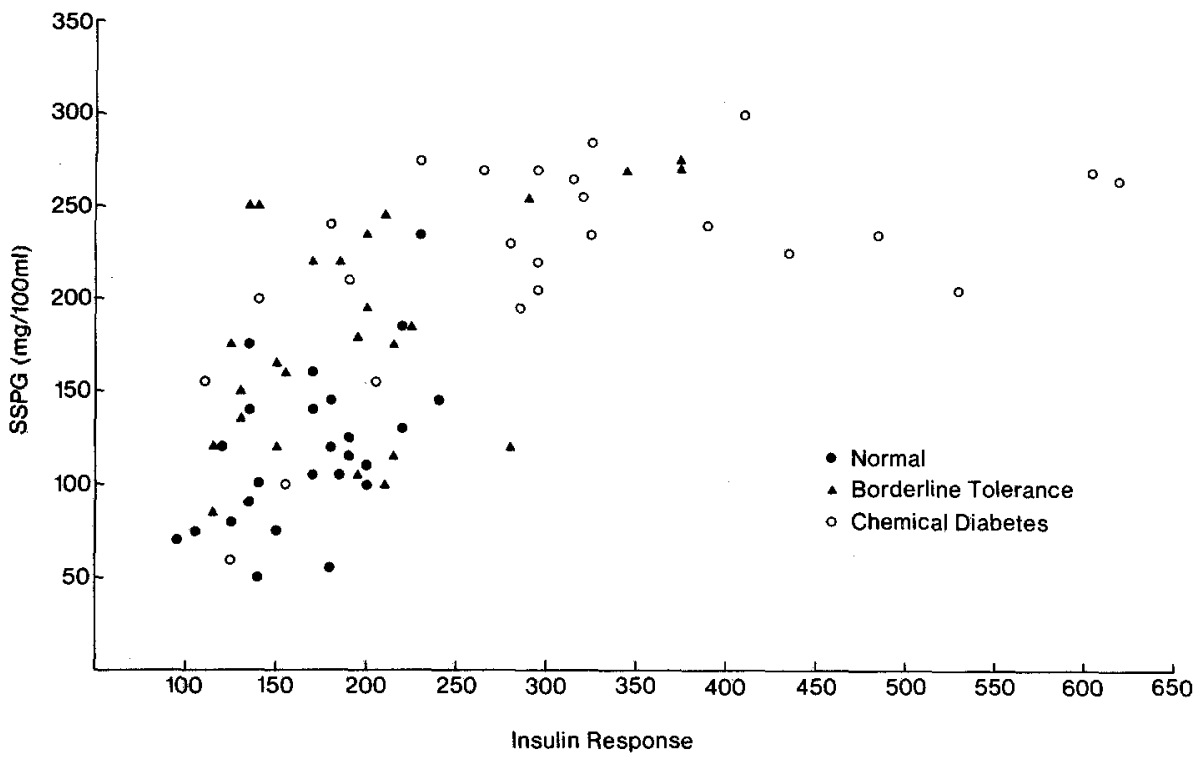

Fig. 4. The relationship between insulin response (area under the plasma insulin curve during the OGTT) and insulin resistance (steady state plasma glucose level during the infusion) in all subjects studied tients with borderline tolerance $(\mathrm{p}<0.01)$. The mean steady state plasma insulin response was virtually identical in the three groups. Thus, the more severe the abnormality of glucose tolerance, the higher the SSPG, i.e., the greater the insulin resistance.

The individual SSPG responses demonstrate that variation also exists in this measurement. The coefficient of variation was $36 \%, 32 \%$ and $25 \%$, for normal subjects, patients with borderline tolerance and chemical diabetics, respectively. However, 19 of 25 patients with chemical diabetes had SSPG responses which were more than two standard deviations above the mean response of control subjects. Thus, it appears that the majority of patients with oral glucose intolerance are more insulin resistant than are the majority of normal subjects. On the other hand, there are certainly some patients with glucose intolerance who appear to be insulin sensitive.

The relationship (Spearman Rank Correlation Coefficient) between insulin response and SSPG for the entire experimental group is presented in Figure 4. A strong correlation $(r=0.64, p<0.001)$ exists between these two variables. These results suggest that the greater the insulin response, the greater the degree of insulin resistance, and this relationship seems to be independent of diagnostic category. On the other hand, certain groupings emerge. For example, the majority of patients with chemical diabetes share a greater insulin response and higher SSPG level, and these two variables seem to clearly separate them from most subjects with normal glucose tolerance. However, a few patients with chemical diabetes have insulin responses and SSPG levels well within the normal range, and they seem to dif- fer, in both respects, from the remainder of the patients with chemical diabetes.

\section{Discussion}

These results confirm earlier studies which indicated that the mean insulin response of patients with mild diabetes mellitus was not less than that of subjects with normal glucose tolerance [5-16]. They also confirm the observation by Fajans and associates of considerable variation in the insulin response to oral glucose among normal subjects and patients with mild diabetes [1]. There is also considerable variation in the relative ability of insulin to promote glucose uptake in these same subjects. However, the degree of variability is not so great as to obscure the fact that most patients with chemical diabetes were more insulin resistant and had, in general, the greatest total insulin response to oral glucose. In this sense, these results differ somewhat from those of Fajans and associates [1], who found that the majority of their patients with chemical diabetes had a somewhat lower insulin response than did normal subjects. The explanation for this difference is not clear, but it may reflect the fact that our control and experimental groups were approximately twice as old as the subjects they studied.

Although these results indicate that there is considerable variation in the insulin response within each of the groups, they do not necessarily mean that these groups are heterogenous. For example, the diagnostic accuracy of the OGTT is not perfect [17], and apparent heterogeneity of the insulin response may be due to the inclusion of individuals 
within the "wrong" diagnostic category. Furthermore, there is considerable variability in the insulin response of any given individual. Thus, the plasma insulin response to two successive oral glucose tolerance tests, performed $48 \mathrm{~h}$ apart, differed by more than $30 \%$ in half the patients studied [18]. In an attempt to distinguish between variability and heterogeneity, we examined the same subjects in terms of another characteristic, i. e., their resistance to insulin-mediated glucose uptake (insulin resistance). The result of this analysis (Fig. 3) indicates that variability of insulin resistance also existed in these subjects. However, it is likely that these two characteristics, i. e., insulin response and insulin resistance, are related [19] and this relationship might help differentiate between heterogeneity as contrasted to variability. Evidence for the fact that the two characteristics are related is presented in Figure 4, which demonstrates a highly significant correlation between height of insulin response and severity of insulin resistance in the study population. Furthermore, when both characteristics are taken into consideration, we feel that there is support for the notion of true heterogeneity in patients with chemical diabetes. Thus, most patients with chemical diabetes can be distinguished from most normal subjects when both insulin response and insulin resistance are taken into consideration. On the other hand, there are some patients, diagnosed as having chemical diabetes, who cannot be differentiated from normal with use of both characteristics.

In light of the above we think that true heterogeneity does exist within the group of patients classified as having chemical diabetes. In most of these patients we believe that the abnormal glucose tolerance test is due to resistance to the action of insulin, and the exaggerated insulin response is an attempt to maintain glucose homeostasis. On the other hand, we think that the glucose intolerance in the patients who demonstrate a low insulin response and normal insulin sensitivity is secondary to deficient insulin secretion.

The division of patients with chemical diabetes into these two subgroups may also help in understanding which of these patients will develop progressive deterioration of glucose tolerance. The majority of patients with chemical diabetes do not experience deterioration of glucose tolerance with time, and this is true irrespective of age $[20,21]$. However, a minority of patients with chemical diabetes do show progressive deterioration and these subjects seem to be those with a diminished insulin response to glucose [22-24]. It is possible that deterioration of glucose tolerance is confined to those subjects whose chemical diabetes is secondary to a diminished insulin response and is associated with a further attenuation of the insulin response. In contrast, the majority of patients currently diagnosed as having chemical diabetes may not be insulin deficient - their glucose intolerance is due to their increased degree of insulin resistance - and these patients rarely develop insulin deficiency or develop glucose intolerance.

In conclusion, these results indicate that considerable variability exists in both plasma insulin response to oral glucose and plasma glucose response to infused insulin in patients with glucose intolerance. A significant relationship was seen between height of insulin response and degree of insulin resistance in the entire population, and the nature of this relationship seems to confirm the suggestion of Fajans et al. [1] that there is heterogeneity within patients with chemical diabetes. The subdivision of chemical diabetics into those patients with both insulin resistance and an increased insulin response, as contrasted to those with a primary defect of insulin relcase, without insulin resistance, seems to account for both the heterogeneity and the clinical course of these patients. Obviously, further study will be needed to evaluate this working hypothesis.

\section{References}

1. Fajans, S.S., Floyd, J.C. Jr., Taylor, C.E., Pek, S.: Heterogeneity of insulin responses in latent diabetes. Trans. Assoc. Am. Phys. 87, 83-94 (1975)

2. Ginsberg, H., Olefsky, J.M., Reaven, G.M.: Further evidence that insulin resistance exists in patients with chemical diabetes. Diabetes 23, 674-678 (1974)

3. Shen, S.-W., Reaven, G. M., Farquhar, J. W.: Comparison of impedance to insulin-mediated glucose uptake in normal subjects and in subjects with latent diabetes. J. Clin. Invest. 49, 2151-2160 (1970)

4. Desbuquois, B., Aurbach, G. D.: Use of polyethylene glycol to separate free and antibody-bound peptide hormones in radioimmunoassays. J. Clin. Endocrinol. Metab. 33, 732-738 (1971)

5. Berson, S. A., Yalow, R.S.: Some current controversies in diabctes research. Diabctes 14, 549-572 (1965)

6. Buchanan, K.D., McKiddie, M.T.: Factors determining the plasma insulin response to oral glucose in diabetes mellitus. Diabetes 16, 466-471 (1967)

7. Seltzer, H.S., Allen, W.D., Herron, A.L.Jr., Brennan, M. T.: Insulin secretion in response to glycemic stimulus: relation of delayed initial rclease to carbohydrate intolerance in mild diabetes. J. Clin. Invest. 46, 323-335 (1967)

8. Reaven, G.M., Miller, R.: Study of the relationship between glucose and insulin responses to an oral glucose load. Diabetes 17, 560-569 (1968)

9. Chiles, R., Tzagournis, M.: Excessive serum insulin response to oral glucose in obesity and mild diabetes. Diabetes $\mathbf{1 9}$, 458-464 (1970)

10. Rosenbloom, A.L.: Insulin responses of children with chemical diabetes mellitus. N. Engl. J. Med. 282, 1228-1231 (1970) 
11. Reaven, G.M., Shen, S.-W., Silvers, A., Farquhar, J. W.: Is there a delay in the plasma insulin response of patients with chemical diabetes? Diabetes 20, 416-423 (1971)

12. Reaven, G.M., Olefsky, J.M., Farquhar, J. W.: Does hyperglycaemia or hyperinsulinaemia characterise the patient with chemical diabetes? Lancet 1972 I, 1247-1249

13. Jackson, W.P. U., van Mieghem, W., Keller, P.: Insulin excess as the initial lesion in diabetes. Lancet 1972 I, 1040-1044

14. Johansen, I.: Normal initial insulin response in mild diabetes. Metabolism 21, 1177-1180 (1972)

15. Duckworth, W. C., Kitabchi, A.E.: Direct measurement of plasma proinsulin in normal and diabetic subjects. Am. J. Med. 53, 418-427 (1972)

16. Savage, P.J., Dippe, S. E., Bennett, P.H., Gorden, P., Roth, J., Rushforth, N. B., Miller, M.: Hyperinsulinemia and hypoinsulinemia. Insulin responses to oral carbohydrate over a wide spectrum of glucose tolerance. Diabetes 24, 362-368 (1975)

17. Olefsky, J.M., Farquhar, J.W., Reaven, G.M.: Do the oral and intravenous glucose tolerance tests provide similar diagnostic information in patients with chemical diabetes mellitus? Diabetes 22, 202-209 (1973)

18. Olefsky, J.M., Reaven, G.M.: Insulin and glucose responses to identical glucose loads performed forty-eight hours apart. Diabetes 23, 449-453 (1974)

19. Olefsky, J.M., Farquhar, J.W., Reaven, G.M.: Reappraisal of the role of insulin in hypertriglyceridemia. Am. J. Med. 57, 551-560 (1974)

20. O'Sullivan, J.B., Mahan, C.M.: Prospective study of 352 young patients with chemical diabetes. N. Engl. J. Med. 278, 1038-1041 (1968)

21. Rosenbloom, A.L., Drash, A., Guthrie, R.: Conference summary: chemical diabetes in childhood. Metabolism 22, 413-419 (1973)

22. Guthrie, R.A., Guthrie, D.S., Murthy, D.T.N., Jackson, R. L., Lang, J.: Standardization of the oral glucose tolerance test and the criteria for the diagnosis of chemical diabetes in children. Metabolism 22, 275-282 (1973)

23. Pildes, R.S.: Adult onset diabetes mellitus in childhood. Metabolism 22, 307-311 (1973)

24. Rosenbloom, A. L., Wheeler, L., Bianchi, R., Chin, F.T., Tiwary, C. M., Grgic, A.: Age-adjusted analysis of insulin responses during normal and abnormal glucose tolerance tests in children and adolescents. Diabetes 24, 820-828 (1975)

Received: October 10, 1976, and in revised form:

December 22, 1976

Dr. G. M. Reaven

Veterans Administration Hospital

3801 Miranda Avenue

Palo Alto, CA 94304, USA 